\title{
Correlation energy of a homogeneous dipolar Fermi gas
}

\author{
Bo Liu, Lan Yin* \\ School of Physics, Peking University, Beijing 100871, China
}

(Dated: June 20, 2018)

\begin{abstract}
We study the normal state of a $3-d$ homogeneous dipolar Fermi gas beyond the Hartree-Fock approximation. The correlation energy is found of the same order as the Fock energy, unusually strong for a Fermi-liquid system. As a result, the critical density of mechanical collapse is smaller than that estimated in the Hartree-Fock approximation. With the correlation energy included, a new energy functional is proposed for the trapped system, and its property is explored.
\end{abstract}

*Electronic address: yinlan@pku.edu.cn 


\section{INTRODUCTION}

The successful creation of KRb polar molecules has provided a new many-body system to explore [1]. A dipolar Fermi gas is quite different from a typical Fermi gas or an electron gas due to the long-range and anisotropic interaction between the dipoles. Theoretical studies

on various properties of the dipolar Fermi gas have been carried out [2]. The Fermi surface is deformed into an elliptical shape [3] in the normal state of a homogeneous system. A $p$-wave superfluid state was proposed [4]. So far most theoretical studies were done in the Hartree-Fock (HF) approximation which underestimates correlation effects. In this paper, we go beyond the HF approximation to obtain the correlation energy of a homogenous dipolar Fermi gas in the normal state, and use it in the density-functional description of inhomogeneous cases.

This paper is organized as follows. In section II, the correlation energy of a homogeneous dipolar Fermi gas is obtained in the perturbation theory. We find that the ground state energy is lowered by the correlation effect, leading to a significantly smaller critical density of mechanical collapse than the HF result. In section III, the expression of the correlation energy is used to construct the energy functional of a trapped dipolar Fermi gas in the local density approximation. This energy functional is shown to satisfy a virial theorem. The critical total number of polar molecules for mechanical collapse in the trap is obtained. Conclusions are given in section IV.

\section{A HOMOGENEOUS DIPOLAR FERMI GAS}

We consider a homogeneous dipolar Fermi gas with mass $m$ and permanent electric dipole moment $d$ polarized along the $z$-axis. The uniform system is described by the Hamiltonian

$$
H=\sum_{\mathbf{k}} \epsilon_{k} a_{\mathbf{k}}^{\dagger} a_{\mathbf{k}}+\frac{1}{2 V^{2}} \sum_{\mathbf{q} \neq 0} \sum_{\mathbf{k}_{1} \mathbf{k}_{2}} V_{\mathbf{q}} a_{\mathbf{k}_{1}+\mathbf{q}}^{\dagger} a_{\mathbf{k}_{2}-\mathbf{q}}^{\dagger} a_{\mathbf{k}_{2}} a_{\mathbf{k}_{1}},
$$

where $a_{\mathbf{k}}$ and $a_{\mathbf{k}}^{\dagger}$ are the fermion annihilation and creation operators, $\epsilon_{k}=\hbar^{2} k^{2} /(2 m)$ is the fermion kinetic energy, $V_{\mathbf{q}}=4 \pi d^{2}\left(3 \cos ^{2} \phi_{\mathbf{q}}-1\right) / 3$ is the dipole-dipole interaction for the transferred wavevector $\mathbf{q}, \phi_{\mathbf{q}}$ is the angle between the $z$-axis and $\mathbf{q}$, and $V$ is the volume.

In the HF approximation, the single-particle excitation energy and the Fermi surface are no longer isotropic due to the anisotropic dipole-dipole interaction [3], and the HF 
Hamiltonian is given by

$$
H_{H F}=\sum_{\mathbf{k}} \varepsilon_{\mathbf{k}} a_{\mathbf{k}}^{\dagger} a_{\mathbf{k}}-\frac{1}{2 V^{2}} \sum_{\mathbf{k}_{1}, \mathbf{k}_{2}} V_{\mathbf{k}_{1}-\mathbf{k}_{2}} n_{\mathbf{k}_{1}} n_{\mathbf{k}_{2}},
$$

where the single particle energy $\varepsilon_{\mathbf{k}}$ is given by

$$
\varepsilon_{\mathbf{k}}=\epsilon_{k}+\frac{1}{V} \sum_{\mathbf{k}^{\prime}} V_{\mathbf{k}-\mathbf{k}^{\prime}} n_{\mathbf{k}^{\prime}}
$$

$n_{\mathbf{k}}=\theta\left(\mu-\varepsilon_{\mathbf{k}}\right)$ is the occupation number, and the chemical potential $\mu$ is determined from the total number constraint $N=\sum_{\mathbf{k}} n_{\mathbf{k}}$. In the HF approximation, the occupation number $n_{\mathbf{k}}$ can be solved self consistently. To the first order in the interaction strength, the deformed Fermi surface is given by [5]

$$
k(\phi)=k_{F}+\frac{1}{9 \pi} \frac{m d^{2} k_{F}^{2}}{\hbar^{2}}\left(3 \cos ^{2} \phi-1\right),
$$

where $k_{F}$ is the Fermi wavevector of the noninteracting Fermi gas. To the first order, this deformation does not change the volume inside the Fermi surface, nor the total energy. The HF approximation modifies the ground state energy starting only in the second order [5],

$$
\frac{E_{H F}}{V}=\frac{\hbar^{2} k_{F}^{5}}{m}\left[\frac{1}{20 \pi^{2}}-\frac{1}{405 \pi^{4}}\left(\frac{m d^{2} k_{F}}{\hbar^{2}}\right)^{2}\right]
$$

or in terms of the Fermion density $n$,

$$
\frac{E_{H F}}{V}=\frac{\hbar^{2}}{m}\left[\frac{3}{10}\left(6 \pi^{2}\right)^{\frac{2}{3}} n^{\frac{5}{3}}-\frac{4}{45}\left(6 \pi^{2}\right)^{\frac{1}{3}}\left(\frac{m d^{2}}{\hbar^{2}}\right)^{2} n^{\frac{7}{3}}\right]
$$

where $n$ is the Fermion density in real space.

Although the HF approximation is accurate for the ground state energy in the first order, in general it is insufficient for the second or higher-order ground state energy. Kohn and Luttinger [6] showed that in Fermi systems with anisotropic Fermi surfaces due to interaction, the second-order ground state energy consists of two parts, one from the HF approximation and the other from the Brueckner-Goldstone (BG) formula. The BG formalism is the standard perturbation theory which was used by Lee and Yang to obtain the second-order ground state energy of a dilute Fermi gas [7]. In the BG formalism, the second-order contribution to the ground-state energy of a dipolar Fermi gas is given by

$$
E_{B G}^{(2)}=\sum_{m \neq 0} \frac{\left\langle 0\left|H_{1}\right| m\right\rangle\left\langle m\left|H_{1}\right| 0\right\rangle}{E_{0}-E_{m}},
$$


where $|0\rangle$ and $|m\rangle$ are the ground and excited states of the noninteracting Fermi gas with eigenenergies $E_{0}$ and $E_{m}$, and $H_{1}$ is the dipole-dipole interaction given by the second r.-h.-s. term in Eq. (1). This BG energy can be further written as

$$
\begin{aligned}
\frac{E_{B G}^{(2)}}{V}= & \frac{1}{2 V^{3}} \sum_{\mathbf{k}_{1}, \mathbf{k}_{2}, \mathbf{q}} \frac{V_{\mathbf{q}}\left(V_{\mathbf{q}}-V_{\mathbf{k}_{2}-\mathbf{k}_{1}-\mathbf{q}}\right)}{\epsilon_{\mathbf{k}_{1}}+\epsilon_{\mathbf{k}_{2}}-\epsilon_{\mathbf{k}_{1}+\mathbf{q}}-\epsilon_{\mathbf{k}_{2}-\mathbf{q}}} \\
& \times f_{\mathbf{k}_{1}} f_{\mathbf{k}_{2}}\left(1-f_{\mathbf{k}_{1}+\mathbf{q}}\right)\left(1-f_{\mathbf{k}_{2}-\mathbf{q}}\right),
\end{aligned}
$$

where $f_{\mathbf{k}}=\theta\left(k_{F}-k\right)$ is the fermion occupation number in the noninteracting ground state $|0\rangle$. The k-space summations in Eq. (6) can be rescaled into integrals over dimensionless variables,

$$
\begin{aligned}
\frac{E_{B G}^{(2)}}{V}= & \frac{m d^{4} k_{F}^{7}}{32 \pi^{7} \hbar^{2}} \int d^{3} x_{1} d^{3} x_{2} d^{3} y \frac{\left(\cos ^{2} \phi_{\mathbf{y}}-\frac{1}{3}\right)\left(\cos ^{2} \phi_{\mathbf{y}}-\cos ^{2} \phi_{\mathbf{x}_{2}-\mathbf{x}_{1}-\mathbf{y}}\right)}{x_{1}^{2}+x_{2}^{2}-\left|\mathbf{x}_{\mathbf{1}}+\mathbf{y}\right|^{2}-\left|\mathbf{x}_{\mathbf{2}}-\mathbf{y}\right|^{2}} \\
& \times \theta\left(1-x_{1}\right) \theta\left(1-x_{2}\right) \theta\left(\left|\mathbf{x}_{\mathbf{1}}+\mathbf{y}\right|-1\right) \theta\left(\left|\mathbf{x}_{\mathbf{2}}-\mathbf{y}\right|-1\right)
\end{aligned}
$$

where $\mathbf{x}_{i}=\mathbf{k}_{i} / k_{F}$ and $\mathbf{y}=\mathbf{q} / k_{F}$. Eq. (17) indicates that the BG energy density is proportional to $d^{4} n^{7 / 3}$. We obtain the coefficient by Monte Carlo integration [8],

$$
\frac{E_{B G}^{(2)}}{V}=-0.66 \frac{m d^{4}}{\hbar^{2}} n^{\frac{7}{3}} .
$$

By definition, the correlation energy is the difference between the true ground state energy and the ground state energy in the HF approximation. Therefore in the second order, the correlation energy of a dipolar Fermi gas is given by Eq. (8). Its negative sign indicates that the ground state energy is lowered by the correlation effect.

The total ground-state energy of a homogeneous dipolar Fermi gas up to the second order can be obtained by adding Eq. (8) to Eq. (44),

$$
\frac{E}{V}=\frac{\hbar^{2}}{m}\left[\frac{3}{10}\left(6 \pi^{2}\right)^{\frac{2}{3}} n^{\frac{5}{3}}-1.01\left(\frac{m d^{2}}{\hbar^{2}}\right)^{2} n^{\frac{7}{3}}\right] .
$$

Since the second r.-h.-s. term in Eq. (9) is negative, and its magnitude increases faster than the kinetic energy when the density increases, there exists a critical density $n_{c}$ for mechanical collapse, $(\partial \mu / \partial n)_{n_{c}}=0$, where the chemical potential $\mu$ is given by

$$
\mu=\frac{\partial E}{\partial N}=\frac{\hbar^{2}}{m}\left[\frac{1}{2}\left(6 \pi^{2}\right)^{\frac{2}{3}} n^{\frac{2}{3}}-2.36\left(\frac{m d^{2}}{\hbar^{2}}\right)^{2} n^{\frac{4}{3}}\right] .
$$

As shown in Fig. 1, the chemical potential reaches the maximum at the critical density $n_{c}=2.04\left(\hbar^{2} / m d^{2}\right)^{3}$, whereas the simple HF result implies $n_{c}=9.97\left[\hbar^{2} /\left(m d^{2}\right)\right]^{3}$. Beyond 


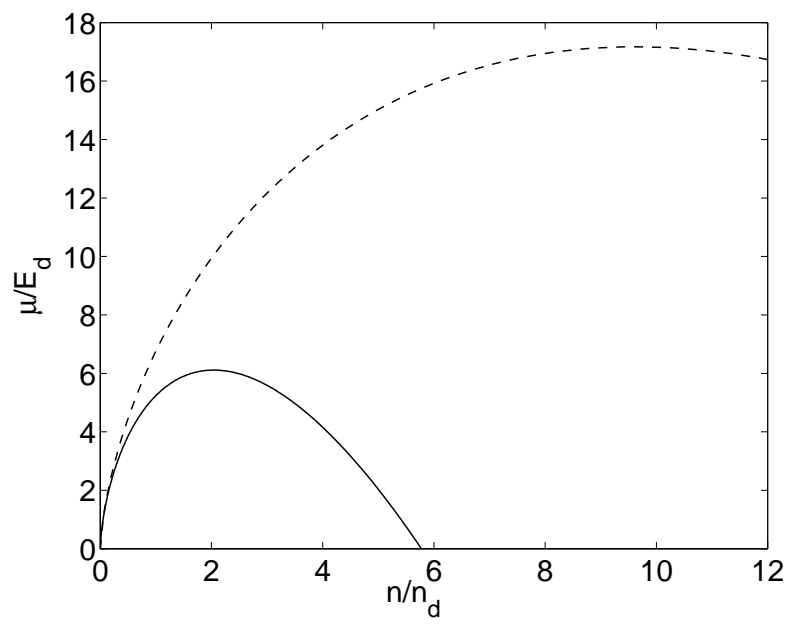

FIG. 1: Chemical potential $\mu$ as the function of the fermion density $n$, where the unit of $\mu$ is $E_{d} \equiv \hbar^{6} /\left(m^{3} d^{4}\right)$ and the unit of $n$ is $n_{d} \equiv\left[\hbar^{2} /\left(m d^{2}\right)\right]^{3}$. The solid and dashed lines are the chemical potentials with and without the contribution from correlation energy respectively.

this critical density $n_{c}$, the compressibility turns negative and the system is no longer stable against mechanical collapse. We should point out that the perturbation method is valid when the absolute value of the interaction energy is smaller than the kinetic energy, $n<$ 9.58( $\left.\hbar^{2} / m d^{2}\right)^{3}$ as indicated by Eq. (9). At $n_{c}=2.04\left(\hbar^{2} / m d^{2}\right)^{3}$, the perturbation still works, but higher order terms may be needed to produce more quantitatively accurate results. Nonetheless, the correlation energy effectively makes the system more vulnerable towards mechanical collapse.

In the experiment on KRb molecules, the dipole moment for the singlet rovibrational ground state is $d=0.566(17)$ Debye [9]. Our calculation suggests that the critical density for mechanical collapse in this case is approximately $n_{c} \simeq 9.23 \times 10^{12} \mathrm{~cm}^{-3}$. In other references, this critical density is estimated to be $1.48 \times 10^{14} \mathrm{~cm}^{-3}$ in the variational approach [10], $4.89 \times 10^{12} \mathrm{~cm}^{-3}$ from the zero-sound analysis [5], and $4.80 \times 10^{12} \mathrm{~cm}^{-3}$ in the collective-mode study [11]. The highest molecule density in the current experiment is about $0.3 \times 10^{12} \mathrm{~cm}^{-3}$ [12] when the temperature is at 1.4 times the Fermi temperature. It is hopeful that these theoretical results can be tested in future experiments with higher molecule density achieved. 


\section{TRAPPED DIPOLAR FERMI GAS}

The Hamiltonian of a dipolar Fermi gas in a harmonic trap is given by

$$
\begin{aligned}
H & =\int d^{3} r \psi^{\dagger}(\mathbf{r})\left[\frac{-\hbar^{2} \nabla^{2}}{2 m}+U_{h o}\right] \psi(\mathbf{r}) \\
& +\frac{1}{2} \iint d^{3} r d^{3} r^{\prime} \psi^{\dagger}(\mathbf{r}) \psi^{\dagger}\left(\mathbf{r}^{\prime}\right) V_{d d}\left(\mathbf{r}-\mathbf{r}^{\prime}\right) \psi\left(\mathbf{r}^{\prime}\right) \psi(\mathbf{r})
\end{aligned}
$$

where the dipole-dipole interaction $V_{d d}(\mathbf{r})$ is given by $V_{d d}(\mathbf{r})=\left(d^{2} / r^{3}\right)\left[1-3\left(z^{2} / r^{2}\right)\right]$, and $\psi(\mathbf{r})$ is the fermion field operator. In the following we consider an axially symmetric trap with the trapping potential given by $U_{h o}=m\left(\omega_{\rho}^{2} x^{2}+\omega_{\rho}^{2} y^{2}+\omega_{z}^{2} z^{2}\right) / 2$ and the trap aspect ratio given by $\lambda \equiv \omega_{z} / \omega_{\rho}$.

We consider the case that the scale of the trap is much larger than the interparticle distance, so the local density approximation (LDA) can be applied. The total energy $E$ can be written as

$$
E=E_{k i n}+E_{h o}+E_{H a r}+E_{s}
$$

where $E_{k i n}, E_{h o}$, and $E_{H a r}$ are kinetic, trap, and Hartree energies. The energy $E_{s}$ includes both the second-order Fock and correlation energies. Density functionals of these energies are given by

$$
\begin{aligned}
E_{k i n} & =\frac{3(6 \pi)^{\frac{2}{3}} \hbar^{2}}{10 m} \int d^{3} r n^{\frac{5}{3}}(\mathbf{r}), \\
E_{h o} & =\int d^{3} r U_{h o}(\mathbf{r}) n(\mathbf{r}), \\
E_{\text {Har }} & =\frac{1}{2} \iint d^{3} r d^{3} r^{\prime} n(\mathbf{r}) V_{d d}\left(\mathbf{r}-\mathbf{r}^{\prime}\right) n\left(\mathbf{r}^{\prime}\right), \\
E_{s} & =-1.01 \frac{m d^{4}}{\hbar^{2}} \int d^{3} r n^{\frac{7}{3}}(\mathbf{r})
\end{aligned}
$$

where $n(\mathbf{r})$ is the fermion density with the number constraint $N=\int d^{3} r n(\mathbf{r})$. At the ground state, the fermion density $n(\mathbf{r})$ satisfies the energy-extreme condition,

$$
\frac{\delta E}{\delta n}-\mu \frac{\delta N}{\delta n}=0
$$

from which the chemical potential $\mu=\partial E / \partial N$ can be determined,

$$
\mu=\frac{\hbar^{2}}{2 m}\left(6 \pi^{2}\right)^{\frac{2}{3}} n^{\frac{2}{3}}(\mathbf{r})+\frac{\hbar \omega_{\rho}}{2}\left(x^{2}+y^{2}+\lambda^{2} z^{2}\right)
$$




$$
\begin{aligned}
& +d^{2} \int d^{3} r^{\prime} \frac{1-3 \cos ^{2} \theta_{\mathbf{r}-\mathbf{r}^{\prime}}}{\left|\mathbf{r}-\mathbf{r}^{\prime}\right|^{3}} n\left(\mathbf{r}^{\prime}\right) \\
& -2.36 \frac{m d^{4}}{\hbar^{2}} n^{\frac{4}{3}}(\mathbf{r}) .
\end{aligned}
$$

In LDA, the total energy $E$ satisfies a virial theorem which can be proved by the scaling transformation of the density [13, 14$]$

$$
n(\mathbf{r}) \rightarrow \eta^{3+\alpha} n(\eta \mathbf{r})
$$

where $\eta$ and $\alpha$ are general numbers. Under this transformation, the total density and various parts of the total energy $E$ transform as

$$
\begin{aligned}
N & \rightarrow \eta^{\alpha} N, \\
E_{k i n} & \rightarrow \eta^{2+\frac{5}{3} \alpha} E_{k i n}, \\
E_{h o} & \rightarrow \eta^{-2+\alpha} E_{h o}, \\
E_{H a r} & \rightarrow \eta^{3+2 \alpha} E_{H a r}, \\
E_{s} & \rightarrow \eta^{4+\frac{7}{3} \alpha} E_{s} .
\end{aligned}
$$

Close to $\eta=1$, to the first order in $\delta \eta=\eta-1$, the changes in these quantities are given by

$$
\begin{aligned}
\delta N & =\alpha \delta \eta N \\
\delta E_{k i n} & =\left(2+\frac{5}{3} \alpha\right) \delta \eta E_{k i n} \\
\delta E_{h o} & =(-2+\alpha) \delta \eta E_{h o} \\
\delta E_{H a r} & =(3+2 \alpha) \delta \eta E_{H a r} \\
\delta E_{s} & =\left(4+\frac{7}{3} \alpha\right) \delta \eta E_{c} .
\end{aligned}
$$

Therefore the change in the total energy is given by

$$
\delta E=\delta E_{k i n}+\delta E_{h o}+\delta E_{H a r}+\delta E_{s},
$$

and from the energy-extreme condition Eq. (12) it also satisfies the equation

$$
\delta E=\frac{\partial E}{\partial N} \delta N
$$

From the above equations, we have

$$
\begin{aligned}
\alpha N \frac{\partial E}{\partial N} & =\left(2+\frac{5}{3} \alpha\right) E_{k i n}+(-2+\alpha) E_{h o} \\
& +(3+2 \alpha) E_{H a r}+\left(4+\frac{7}{3} \alpha\right) E_{s}
\end{aligned}
$$


which leads to two independent equations

$$
\begin{aligned}
& 2 E_{k i n}-2 E_{h o}+3 E_{\text {Har }}+4 E_{s}=0 \\
& \frac{5}{3} E_{k i n}+E_{h o}+2 E_{H a r}+\frac{7}{3} E_{s}=N \frac{\partial E}{\partial N} .
\end{aligned}
$$

Eqs. (18) and (19) together with identities

$$
\begin{aligned}
d \frac{\partial E}{\partial d} & =2 E_{\text {Har }}+4 E_{s}, \\
\omega_{\rho} \frac{\partial E}{\partial \omega_{\rho}} & =2 E_{h o} \\
m \frac{\partial E}{\partial m} & =E_{h o}-E_{k i n}+E_{s}
\end{aligned}
$$

yield

$$
\begin{aligned}
d \frac{\partial E}{\partial d} & =\frac{4}{3} m \frac{\partial E}{\partial m} \\
\omega_{\rho} \frac{\partial E}{\partial \omega_{\rho}} & =E+\frac{1}{3} m \frac{\partial E}{\partial m} \\
N \frac{\partial E}{\partial N} & =\frac{4}{3} E+\frac{1}{9} m \frac{\partial E}{\partial m}
\end{aligned}
$$

indicating the total energy $E$ of the form

$$
E\left(m, \omega_{\rho}, d, N\right)=\hbar \omega_{\rho} N^{\frac{4}{3}} g\left(N^{\frac{1}{6}} C_{d d}\right)
$$

where $C_{d d}$ is the dimensionless dipolar interaction strength, $C_{d d} \equiv m d^{2} / a_{0} \hbar^{2}, a_{0}=\sqrt{\hbar / m \omega_{\rho}}$, and $g(x)$ is a general functional of the variable $x$.

In a trap, the system also suffers mechanical collapse if the total number of fermions exceeds a critical value. In the experiment on KRb polar molecules [9], the optical trapping frequency for the polar molecule is about $\omega_{\rho} /(2 \pi)=109 \mathrm{~Hz}$ and the permanent electric dipole moments measured with stark spectroscopy is $0.566(17)$ Debye for the singlet rovibrational ground state, corresponding to dimensionless dipolar interaction strength $C_{d d}=0.2844$. Our numerical results for the experimental condition show that the critical total number of polar molecules depends on the shape of the optical trap. As shown in Fig. (2), when $\omega_{z}$ is fixed and $\lambda$ varies, the critical total number of polar molecules monotonically increases with the trap aspect ratio $\lambda$, indicating that a more pronounced pancake shape of the trap can help stabilize the dipolar Fermi gas. Our results show that the critical molecule number for mechanical collapse is about $1.75 \times 10^{4}$ for $\lambda=1$, slightly smaller than the HF result 


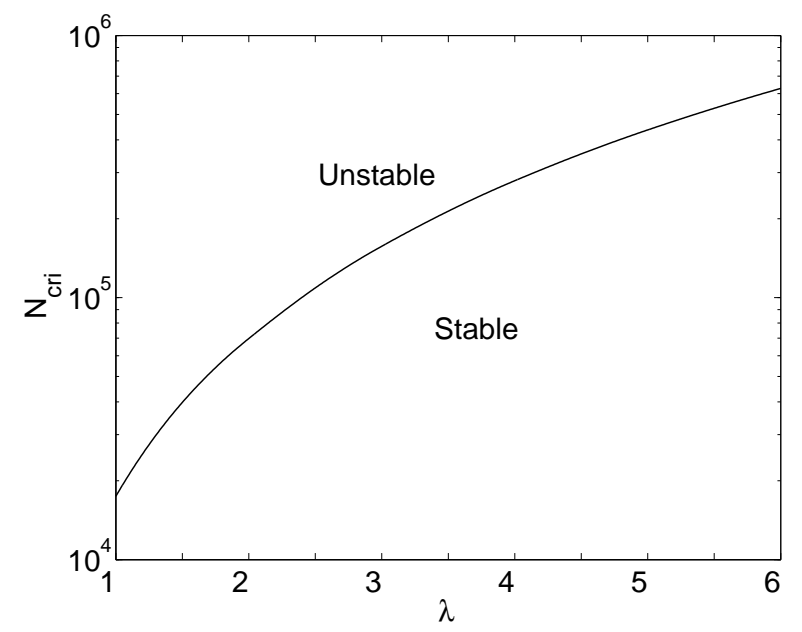

FIG. 2: The critical total number $N_{c r i}$ of polar molecules for mechanical collapse versus the trap aspect ratio $\lambda$ for the singlet rovibrational ground state of KRb with fixed longitudinal trap frequency $\omega_{z} /(2 \pi)=109 \mathrm{~Hz}$.

$2.15 \times 10^{4}[15]$, implying the correlation effect further destabilizes a trapped dipolar Fermi gas. Again we would like to make a remark that our result about the correlation energy given by Eq. (8) is obtained in the second-order perturbation theory. Better results about the correlation energy are needed to estimate the critical molecule number for mechanical collapse with more accuracy.

\section{CONCLUSION}

We studied the normal state of a dipolar Fermi gas beyond the HF approximation. For the homogeneous system, we obtained the ground-state energy including the correlation energy up to second order in the dipole interaction strength, which leads to a critical density for mechanical collapse smaller than that estimated in the HF approximation. For the trapped dipolar Fermi gas, we proposed a energy functional to describe this system based on LDA. We show that this energy functional satisfies a virial theorem. The instability of this system is also investigated. In both cases, the correlation energy makes the system more vulnerable toward mechanical collapse. 


\section{Acknowledgment}

We would like to thank T.-L. Ho, S. Yi, and H. Zhai for helpful discussions and other colleagues for constructive feedbacks. This work is supported by NSFC under Grant No. 10974004, and by Chinese MOST under grant number 2006CB921402.

\section{REFERENCES}

[1] L. D. Carr, D. DeMille, R. V. Krems, and J. Ye, New. J. Phys. 11, 055049 (2009).

[2] M. Baranov, Phys. Rep. 464, 71 (2008).

[3] T. Miyakawa, T. Sogo, and H. Pu, Phys. Rev. A 77061603 (2008).

[4] L. You and M. Marinescu, Phys. Rev. A 60, 2324 (1999).

[5] S. Ronen and J. L. Bohn, Phys. Rev. A 81, 033601 (2010).

[6] W. Kohn and J. M. Luttinger, Phys. Rev. 118, 41 (1960).

[7] T. D. Lee and C. N. Yang, Phys. Rev. 105, 1119 (1957).

[8] W. H. Press, S. A. Teukolsky, W. T. Vetterling, and B. P. Flannery, Numerical Recipes in C (Cambridge, NewYork, 1986).

[9] K. K. Ni, S. Ospelkaus, M. H. G. de Miranda et al, Science 322, 231(2008).

[10] T. Sogo, L. He, T. Miyakawa, S. Yi, H. Lu, and H. Pu, New. J. Phys. 11, 055017 (2009).

[11] C.-K. Chan, C. Wu, W.-C. Lee, and S. Das Sarma, Phys. Rev. A 81, 023602 (2010).

[12] K. K. Ni, S. Ospelkaus, D. Wang et al, Nature 464, 1324(2010).

[13] K. Góral, B.-G. Englert, and K. Rzażewski, Phys. Rev. A 63, 033606 (2001).

[14] K. Góral, M. Brewczyk, and K. Rzażewski, Phys. Rev. A 67, 025601 (2003).

[15] J.-N. Zhang and S. Yi, Phys. Rev. A 80, 053614 (2009). 